\title{
Tullock Contests May Be Revenue Superior to Auctions in a Symmetric Setting
}

\author{
Alexander Matros* \\ University of South Carolina and \\ Alex Possajennikov ${ }^{\dagger}$ \\ University of Nottingham \\ Lancaster University \\ Final version accepted for Economics Letters, March 2016 \\ Published version available at \\ http://dx.doi.org/10.1016/j.econlet.2016.03.003
}

\begin{abstract}
We consider a symmetric two-player common-value setting where each player gets a private signal about the object value. We show that for some parameter values the equilibrium revenue can be higher in a Tullock contest than in the standard auctions.
\end{abstract}

Keywords: common value; contests; auctions

JEL Codes: D82; D44

\footnotetext{
${ }^{*}$ Darla Moore School of Business, University of South Carolina, USA and Lancaster University Management School, Lancaster, UK. Email: alexander.matros@gmail.com

${ }^{\dagger}$ Corresponding author. School of Economics, University of Nottingham, University Park, Nottingham NG7 2RD, United Kingdom. Tel. +44 115 9515461; Fax: +44 115 9514159; Email: alex.possajennikov@nottingham.ac.uk
} 


\section{Introduction}

Economics literature often considers the problem of allocating an indivisible object among individuals. Typical mechanisms for such an allocation are (deterministic) auctions, but an object can also be allocated using a stochastic mechanism. One of the most common stochastic mechanisms is a Tullock contest (Tullock, 1980).

It is often the case in a symmetric setting that the seller's revenue is higher in the standard auctions than in a contest. For example, if an object with a common and known value is allocated between two players, the seller's revenue is equal to the object's value in a standard auction, while it is only one-half of the value in the simplest Tullock contest ("lottery", where the probability of winning the object for a player is the ratio of her bid to the sum of all bids). ${ }^{1}$ On the other hand, Fang (2002) and Epstein et al. (2013) have shown that a contest can achieve a higher revenue than an all-pay auction in asymmetric settings; Franke et al. (2014) further argue that the presence of more than two players increases the chances of contests to achieve a higher revenue. In this paper, we show that a Tullock contest can generate a higher expected revenue even in an ex-ante symmetric setting with two players.

We consider a common-value setting where each player gets a private signal about the object value. Very little is known about revenue comparison of auctions and contests in situations where players have private information, because these situations are typically intractable for contests. In this paper we analyze the Tullock contest and the standard (first-price, second-price and all-pay) auction mechanisms in a symmetric two-player common-value setting where both contest and auction equilibria could be explicitly derived and compared. In our setting, each player receives an independent signal and the common value of the object is an increasing function of both players' signals. The setting is similar to Klemperer's (1998) "wallet game", in which the common value is the sum of the signals of the two players. ${ }^{2}$

In this setting, we derive the equilibrium for the Tullock contest. For the standard auctions, we use, with minor modifications, the available results from the liter-

\footnotetext{
${ }^{1}$ Although in a different setting, Wärneryd (2012, p. 278) also writes that "[f]rom the standpoint of a seller offering a good in an auction a perfectly discriminating mechanism is optimal ...".

${ }^{2}$ Klemperer considers only the second-price auction in his paper.
} 
ature. Although generally there is no clear ranking of auctions in terms of revenue in common-value settings (see Milgrom and Weber, 1982 and Malueg and Orzach, 2009, 2012), in our setting with independent signals the seller's revenue is the same in all three auction mechanisms. Comparing this revenue with the one from the contest, we identify parameter values for which the expected revenue in the contest is higher than in any of the auctions.

\section{Setting: Private Signals and Common Value}

There is an object for sale and there are two risk-neutral buyers. Each of the two buyers gets a private signal about the value of the object: Buyer 1 receives signal $s_{1}$ and Buyer 2 gets signal $s_{2}$. Suppose that the signals have the following structure: each signal is either $H$ (high) with probability $p \in[0,1]$, or $L$ (low, $L<H$ ) with probability $1-p$, independently of the other signal. That is,

$$
s_{i}= \begin{cases}H, & \text { with probability } p \\ L, & \text { with probability } 1-p .\end{cases}
$$

The common value of the object is a function of the two private signals, $v=g\left(s_{1}, s_{2}\right)$. This function satisfies $g(L, L)=0, g(L, H)=g(H, L)=V>0$ and $g(H, H)=(1+$ $\alpha) V$, for $\alpha \geq 0$. The parameter $\alpha$ captures possible nonlinearity or complementarity in the signals.

Two risk-neutral buyers make bids and the object is allocated to one of them according to some mechanism. We first consider the mechanism where the object is allocated according to the Tullock contest and then the standard (all-pay, first-price, and second-price) auction mechanisms.

\section{$2.1 \quad$ Tullock contest}

In the contest, if the bids of the two buyers are $x_{i}$ and $x_{j}$, then buyer $i$ wins the object with probability $x_{i} /\left(x_{i}+x_{j}\right), j \neq i$. A pure strategy $x^{i}=\left(x_{L}^{i}, x_{H}^{i}\right)$ of buyer $i$ consists of two bids, $x_{L}^{i}$ if her signal is $L$ and $x_{H}^{i}$ if her signal is $H$. The expected 
payoff of buyer $i$, conditional on the received signal, is

$$
\begin{aligned}
u_{i}\left(x_{L}^{i}, x^{j} \mid s_{i}=L\right) & =(1-p) \frac{x_{L}^{i}}{x_{L}^{i}+x_{L}^{j}} \times 0+p \frac{x_{L}^{i}}{x_{L}^{i}+x_{H}^{j}} \times V-x_{L}^{i} \\
u_{i}\left(x_{H}^{i}, x^{j} \mid s_{i}=H\right) & =(1-p) \frac{x_{H}^{i}}{x_{H}^{i}+x_{L}^{j}} \times V+p \frac{x_{H}^{i}}{x_{H}^{i}+x_{H}^{j}} \times(1+\alpha) V-x_{H}^{i} .
\end{aligned}
$$

We denote this game by $\mathcal{L}$.

Proposition 1 In the unique symmetric pure-strategy equilibrium of the contest game $\mathcal{L}$, equilibrium bids $x_{L}^{i}=x_{L}^{j}=x_{L}$ and $x_{H}^{i}=x_{H}^{j}=x_{H}$ are

$$
x_{L}= \begin{cases}\frac{1}{4} p V(1-p+D(p, \alpha))(1+p-D(p, \alpha)), & \text { if } 0 \leq \alpha \leq 3, \\ 0, & \text { if } \alpha>3,\end{cases}
$$

and

$$
x_{H}= \begin{cases}\frac{1}{4} p V(1-p+D(p, \alpha))^{2}, & \text { if } 0 \leq \alpha \leq 3 \\ \frac{1}{4} p(1+\alpha) V, & \text { if } \alpha>3\end{cases}
$$

where

$$
D(p, \alpha)=\sqrt{1-p+p^{2}+\alpha p}
$$

Proof. Buyer $i$ maximizes her expected payoffs $(1)-(2)$. Then, the first order conditions are

$$
\begin{aligned}
p \frac{x_{H}^{j}}{\left(x_{L}^{i}+x_{H}^{j}\right)^{2}} V-1 & =0 \\
(1-p) \frac{x_{L}^{j}}{\left(x_{H}^{i}+x_{L}^{j}\right)^{2}} V+p \frac{x_{H}^{j}}{\left(x_{H}^{i}+x_{H}^{j}\right)^{2}}(1+\alpha) V-1 & =0 .
\end{aligned}
$$

The second order conditions are satisfied as the left-hand sides of the above expressions are decreasing in $x_{L}^{i}$ and $x_{H}^{i}$ respectively.

In a symmetric equilibrium, $x_{L}^{j}=x_{L}^{i}=x_{L}$ and $x_{H}^{j}=x_{H}^{i}=x_{H}$. From equation (5), $x_{L}=\sqrt{p V x_{H}}-x_{H}$. Equation (6) becomes

$$
-4 x_{H}+4(1-p) \sqrt{p V} \sqrt{x_{H}}+(1+\alpha) p^{2} V=0
$$


The unique positive solution of this quadratic equation is

$$
x_{H}=\frac{1}{4} p V(1-p+D(p, \alpha))^{2}
$$

where $D(p, \alpha)=\sqrt{1-p+p^{2}+\alpha p}$. Then,

$$
x_{L}=\frac{1}{4} p V(1-p+D(p, \alpha))(1+p-D(p, \alpha)) .
$$

This last expression becomes negative for $\alpha>3$. Thus, the previous derivations hold for $\alpha \leq 3$. For $\alpha>3, x_{L}^{i}=x_{L}^{j}=x_{L}=0$ and $x_{H}^{i}=x_{H}^{j}=x_{H}=p(1+\alpha) V / 4$.

The ex-ante expected revenue in the equilibrium of the contest game is

$$
\pi^{\mathcal{L}}(p, \alpha)=(1-p)^{2} \cdot 2 x_{L}+2(1-p) p \cdot\left(x_{L}+x_{H}\right)+p^{2} \cdot 2 x_{H}=2\left((1-p) x_{L}+p x_{H}\right) .
$$

From Proposition 1, we get

Proposition 2 In the equilibrium of the contest game $\mathcal{L}$, the ex-ante expected revenue is

$$
\pi^{\mathcal{L}}(p, \alpha)= \begin{cases}\frac{1}{2} p V(1-p+D(p, \alpha))\left(1+p-2 p^{2}+(2 p-1) D(p, \alpha)\right), & \text { if } 0 \leq \alpha \leq 3 \\ \frac{1}{2} p^{2}(1+\alpha) V, & \text { if } \alpha>3\end{cases}
$$

\section{$2.2 \quad$ Standard auctions}

In an auction, the highest bid wins the object for sure. We denote by $\mathcal{A}$ any standard (all-pay, first-price, second-price) auction. In general, the revenue equivalence does not hold in common-value auctions (Milgrom and Weber, 1982). However, in our setting with independent signals it does. We present the equilibrium derivations for completeness since they illustrate the reason why the auction revenue may be lower than the contest revenue.

Proposition 3 The ex-ante expected revenue in the symmetric equilibria of the allpay, first-price, and second-price auctions is the same and equal to

$$
\pi^{\mathcal{A}}(p, \alpha)=p^{2}(1+\alpha) V
$$


Proof. We consider the three auctions in turn. As in the contest, buyer $i$ 's auction strategy consists of two (distributions of) bids $\left(b_{L}^{i}, b_{H}^{i}\right)$, depending on her signal. In a symmetric equilibrium both buyers use the same strategy thus we denote by $b_{L}, b_{H}$ the symmetric equilibrium bids and use the superscript to refer to the auction type being considered.

All-pay auction. Siegel (2014) derives the equilibrium for all-pay auctions with several discrete signals. ${ }^{3}$ Applied to our setting, bids $b_{L}^{A l l}=0$ and $b_{H}^{A l l}$ distributed on the interval $[0, p(1+\alpha) V]$ according to the cumulative distribution function $F_{A l l}(x)=$ $x /(p(1+\alpha) V)$ constitute the unique symmetric equilibrium of the all-pay auction $\mathcal{A}^{A l l}$. The expected bid of a high-signal bidder in the all-pay auction is $E\left[b_{H}^{A l l}\right]=p(1+\alpha) V / 2$. Hence, the ex-ante expected revenue is

$\pi^{A l l}(p, \alpha)=(1-p)^{2}\left(2 b_{L}^{A l l}\right)+2(1-p) p\left(b_{L}^{A l l}+E\left[b_{H}^{A l l}\right]\right)+p^{2}\left(2 E\left[b_{H}^{A l l}\right]\right)=p^{2}(1+\alpha) V$.

First-price auction. Wang (1991) considers first-price auctions with an arbitrary finite number of conditionally independent signals. Adapting his arguments to our setting, bids $b_{L}^{F}=0$ and $b_{H}^{F}$ distributed on the interval $[0, p(1+\alpha) V]$ according to the cumulative distribution function $F_{F}(x)=(1-p) x /(p((1+\alpha) V-x))$ constitute the unique symmetric equilibrium of the first-price auction $\mathcal{A}^{F}$. Since the object is sold with probability one, the ex-ante expected revenue can be calculated as

$$
\pi^{F}(p, \alpha)=E[V]-E\left[u_{1}\right]-E\left[u_{2}\right],
$$

where $u_{1}$ and $u_{2}$ are buyer 1's and 2's equilibrium payoffs. In our case

$$
E[V]=(1-p)^{2} \cdot 0+2 p(1-p) \cdot V+p^{2} \cdot(1+\alpha) V
$$

and

$$
E\left[u_{i}\right]=(1-p) \cdot 0+p(1-p) \cdot V .
$$

Therefore, the ex-ante expected revenue is

$$
\pi^{F}(p, \alpha)=2 p(1-p) V+p^{2}(1+\alpha) V-2 p(1-p) V=p^{2}(1+\alpha) V .
$$

\footnotetext{
${ }^{3}$ Siegel's model has strictly positive values while our model has zero value if both signals are low. Nevertheless, his arguments are straightforward to adapt for our case.
} 
Second-price auction. Klemperer (1998) derives a symmetric equilibrium in a setting similar to ours. Adapting his arguments, bids $b_{L}^{S}=0$ and $b_{H}^{S}=(1+\alpha) V$ constitute the unique symmetric equilibrium of the second-price auction $\mathcal{A}^{S}$. The ex-ante expected revenue in this equilibrium is

$$
\pi^{S}(p, \alpha)=(1-p)^{2} \cdot 0+2 p(1-p) \cdot 0+p^{2} \cdot(1+\alpha) V=p^{2}(1+\alpha) V .
$$

\section{Revenue Comparison}

We show that there is no clear revenue ranking between the Tullock contest and the standard auctions in our setting: for some values of $p$ and $\alpha$, the expected revenue is higher in the auctions but for others it is higher in the contest.

Theorem 1 If $p \in[\widehat{p}, 1]$, then

$$
\pi^{\mathcal{A}}(p, \alpha)>\pi^{\mathcal{L}}(p, \alpha) \text { for any } \alpha \geq 0
$$

If $p \in(0, \widehat{p})$, then

$$
\begin{aligned}
& \pi^{\mathcal{A}}(p, \alpha)>\pi^{\mathcal{L}}(p, \alpha), \quad \text { for any } \alpha>\alpha(p), \\
& \pi^{\mathcal{A}}(p, \alpha)<\pi^{\mathcal{L}}(p, \alpha), \quad \text { for any } \alpha<\alpha(p),
\end{aligned}
$$

where $\widehat{p}=0.1(9-\sqrt{6}) \approx 0.65505$ and $\alpha(p)=\left(20 p^{2}-36 p+15\right) /\left(4 p^{2}-12 p+9\right)$.

Proof. Note that $\pi^{\mathcal{A}}(p, \alpha)>\pi^{\mathcal{L}}(p, \alpha)$ for $\alpha \leq 3$, if and only if

$$
3 \alpha-1-2 p \alpha+6 p-4 p^{2}>4(1-p) \sqrt{1-p+p^{2}+\alpha p} .
$$

The right-hand side of this inequality is always non-negative. The left-hand side is negative if $\alpha<\left(1-6 p+4 p^{2}\right) /(3-2 p)$, in which case $\pi^{\mathcal{A}}(p, \alpha)<\pi^{\mathcal{L}}(p, \alpha)$. If the left-hand side of $(10)$ is positive, then $\pi^{\mathcal{A}}(p, \alpha)>\pi^{\mathcal{L}}(p, \alpha)$ if and only if

$$
h(\alpha)=\left(3 \alpha-1-2 p \alpha+6 p-4 p^{2}\right)^{2}-16(1-p)^{2}\left(p \alpha-p+p^{2}+1\right)>0 .
$$

The quadratic equation $h(\alpha)=0$ has two solutions, $\alpha=-1$ and

$$
\alpha=\frac{20 p^{2}-36 p+15}{-12 p+4 p^{2}+9}=\alpha(p) .
$$


Note that $h(\alpha)>0$ if $\alpha<-1$ and $\alpha>\alpha(p)$. Therefore, $\pi^{\mathcal{A}}(p, \alpha)>\pi^{\mathcal{L}}(p, \alpha)$ if and only if $\alpha>\alpha(p)$ for non-negative $\alpha$. Note also that $\alpha(p)>\left(1-6 p+4 p^{2}\right) /(3-2 p)$ for $p \in(0,1)$. Hence, $\pi^{\mathcal{A}}(p, \alpha)<\pi^{\mathcal{L}}(p, \alpha)$ is covered by $\alpha<\alpha(p)$ even if $\alpha<$ $\left(1-6 p+4 p^{2}\right) /(3-2 p)$. If $20 p^{2}-36 p+15<0$, then expression (11) is negative and thus, $\pi^{\mathcal{A}}(p, \alpha)>\pi^{\mathcal{L}}(p, \alpha)$ for any non-negative $\alpha$. Note that $20 p^{2}-36 p+15<0$ if $p \in(0.1(9-\sqrt{6}), 0.1(9+\sqrt{6})) \approx(0.65505,1.1449)$. Therefore, for $p>\hat{p}=0.1(9-\sqrt{6})$ and $p \leq 1$ for any $\alpha \geq 0$ it holds that $\pi^{\mathcal{A}}(p, \alpha)>\pi^{\mathcal{L}}(p, \alpha)$.

For $\alpha>3$ (and thus $\alpha>\alpha(p)$ ), the revenue expressions in (8) - (9) clearly show that $\pi^{\mathcal{A}}(p, \alpha)>\pi^{\mathcal{L}}(p, \alpha)$.

Figure 1 illustrates the values of $p$ and $\alpha$ discussed in Theorem 1 . For small values of $p$ and $\alpha$, the ex-ante expected revenue in the contest is higher than the ex-ante expected auction revenue. This is a surprising result because an auction is typically considered as a mechanism leading to the highest expected revenue, especially in a symmetric setting. The reason for the higher expected revenue in the contest is that the low-signal buyer drops out of the bidding for any values of $p$ and $\alpha$ in a common-value auction in our setting. In the Tullock contest, however, such a buyer has a positive chance of winning by submitting a small positive bid. Although the high-signal buyer bids less in the contest than in an auction, if the probability $p$ that a buyer has a high signal and the complementarity $\alpha$ between the signals are small, then the ex-ante expected revenue from low-signal buyers outweighs the losses from high-signal buyers in the contest. On the other hand, if $p$ and/or $\alpha$ are large enough, then the ex-ante expected revenue is higher in an auction.

\section{Acknowledgments}

We are grateful to an anonymous referee for helpful comments.

\section{References}

Epstein, G.S., Mealem, Y., Nitzan, S. (2013) Lotteries versus all-pay auctions in fair and biased contests. Economics and Politics 25(1), 48-60. 


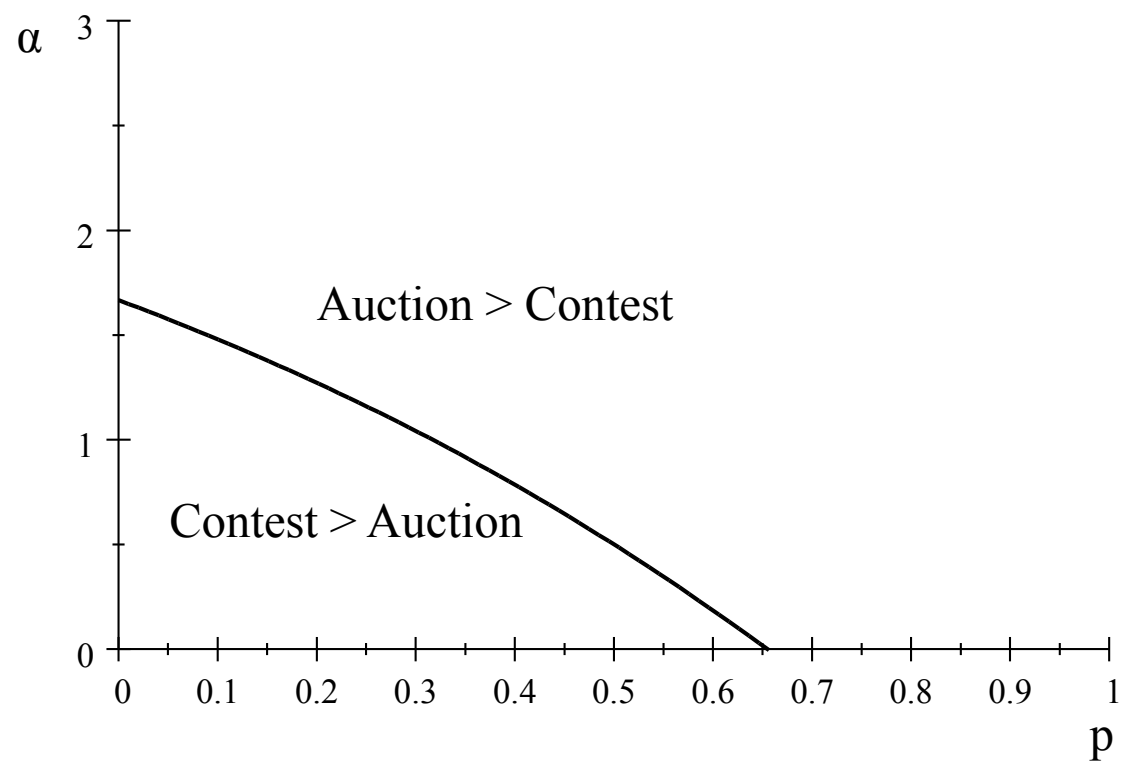

Figure 1: Revenue comparison between the Tullock contest and the auctions

Fang, H. (2002) Lottery versus all-pay auctions models of lobbying. Public Choice 112, 351-371.

Franke, J., Kanzow, C., Leininger, W., Schwartz, A. (2014) Lottery versus all-pay auction contests: a revenue dominance theorem. Games and Economic Behavior 83, 116-126.

Klemperer, P. (1998) Auctions with almost common values: the 'Wallet Game' and its applications. European Economic Review 42, 757-769.

Malueg, D.A., Orzach, R. (2009) Revenue comparison in common-value auctions: Two examples. Economics Letters 105, 177-180.

Malueg, D.A., Orzach, R. (2012) Equilibrium and revenue in a family of commonvalue first-price auctions with differential information. International Journal of Game Theory 41, 219-254. 
Milgrom, P., Weber, R. (1982) A theory of auctions and competitive bidding. Econometrica 50, 1089-1122.

Siegel, R. (2014) Asymmetric all-pay auctions with interdependent valuations. Journal of Economic Theory 153, 684-702.

Tullock, G. (1980) Efficient rent-seeking. In Buchanan, J.M., Tollison, R.D., Tullock, G. (eds) Towards a Theory of Rent-Seeking Society, Texas A\&M University Press, College Station.

Wang, R. (1991) Common-value auctions with discrete private information. Journal of Economic Theory 54, 429-447.

Wärneryd, K. (2012) Multiplayer contests with asymmetric information. Economic Theory 51, 277-287. 